\title{
Quality of Life and Functional Independence in Patients with Osteoarthritis of the Knee
}

\author{
Ivan Luis Andrade Araujo, MSc, Martha Cavalcante Castro, PhD, Carla Daltro, MD, and \\ Marcos Almeida Matos, $\mathrm{PhD}$ \\ Department of Post-Graduation, Bahiana School of Medicine and Public Health, Salvador, Bahia, Brazil
}

\begin{abstract}
Purpose: To verify the association between functional independence (FI) and quality of life (QOL) in patients with knee osteoarthritis.
Materials and Methods: A cross-sectional study composed of 93 patients with knee osteoarthritis was performed. Osteoarthritis was stratified according the classification of Ahlbach. For evaluation of the patient's overall FI, the Barthel index was used. The patient's QOL was measured by means of the Medical Outcomes Study 36-Item Short Form Health Survey (SF-36) questionnaire.

Results: The mean age of the patients was 60 years (range, 54.0 to 69.5 years) and only 32 patients (34.3\%) were found to be independent. Dependent or independent individuals presented difference in all domains of the QOL including physical function ( $\mathrm{p}=0.001)$, role-physical ( $\mathrm{p}=0.005)$, bodily pain $(p=0.001)$, general health $(p=0.004)$, vitality $(p=0.009)$, social function $(p=0.010)$, role-emotional $(p=0.002)$ and mental health $(p=0.001)$. Correlation between FI and QOL was different for all domains of the SF-36. Correlation with FI was strongest for physical function ( $\mathrm{r}=0.609$, $\mathrm{p}<0.001)$, followed by the domains of bodily pain $(\mathrm{r}=0.410, \mathrm{p}<0.001)$ and mental health $(\mathrm{r}=0.402, \mathrm{p}<0.001)$.

Conclusions: Our data demonstrated a strong association of FI with QOL and positive correlations with all QOL domains, indicating the greater the FI is, the higher the QOL is.
\end{abstract}

Keywords: Knee, Osteoarthritis, Function, Quality of life

\section{Introduction}

Degenerative joint disease, also known as osteoarthrosis or osteoarthritis, is one of the most frequent pathological processes of the human species and is considered a growing public health problem $^{1)}$. It is a chronic disease that mainly affects the joints of the knee, hip, hands and spine ${ }^{1,2}$. Osteoarthritis may affect the population in general, however, it presents greater prevalence in women and the elderly ${ }^{1,2)}$. It affects approximately $12 \%$ of the

Received June 3, 2015; Revised (1st) July 9, 2015; (2nd) September 21, 2015; (3rd) November 3, 2015; (4th) November 30, 2015; (5th) January 14, 2016; (6th) January 21, 2016; Accepted January 21, 2016

Correspondence to: Marcos Almeida Matos, $\mathrm{PhD}$

Department of Post-Graduation, Bahiana School of Medicine and Public Health, Rua da Ilha, 378, Itapua, 41620-620 Salvador, Bahia, Brazil

Tel: +55-71-8719-0773, Fax: +55-71-3358-8886

E-mail: marcos.almeida@hotmail.com

This is an Open Access article distributed under the terms of the Creative Commons Attribution Non-Commercial License (http://creativecommons.org/licenses/by-nc/4.0/) which permits unrestricted non-commercial use, distribution, and reproduction in any medium, provided the original work is properly cited. adult population in the US and also around $4 \%$ of the Brazilian population $^{3)}$. The prospects for 2020 are dimmer: osteoarthritis has the potential to transform itself into the 7 th most prevalent disease in the world population ${ }^{4)}$.

The diagnosis, treatment and prognosis of osteoarthritis are at present restricted to clinical evaluations based on signs and symptoms and imaging-based evaluations ${ }^{5}$. This purely medical approach, however, no longer corresponds to the complexity of the impact osteoarthritis has on the health of those who have the disease. Specifically in the knees, the disease is progressive and has the capacity to produce pain, inflammation and joint destruction, with consequent limitations in the range of movement and loss of ability to walk ${ }^{3,5}$. As the disease advances, patients' functional limitations tend to worsen. Therefore, due to limited joint range and pain, daily living activities of these individuals are more compromised, which generates harm in work, leisure and social relationships, resulting in an important decrease in their quality of life (QOL) $)^{3,5-7)}$.

QOL can be evaluated in one of two ways: 1) by means of a generic instrument that would identify the impact of the disease on 
a patient's general health or 2) by means of a specific instrument that would only measure the impact of the disease on the domains of QOL affected directly by the disease itself. Various studies have demonstrated that the loss of QOL of a person with knee osteoarthritis may be a direct consequence of reduced mobility, increased pain, muscle imbalance and restriction of overall functionality ${ }^{5,89}$. However, few studies have sought to determine the impact of a patient's general functional independence (FI) on the general health-related QOL in this important chronic disease ${ }^{9,10)}$.

The Medical Outcomes Study 36-Item Short Form Health Survey (SF-36) is the most commonly used generic instrument for assessing QOL all over the world and the Barthel index is one of the most widely used rating scales for measurement of general FI in patients with neuromuscular and musculoskeletal conditions, as especially recommended for elderly people ${ }^{11,12}$. Therefore, the aim of our study was to verify the association between FI and QOL in patients with knee osteoarthritis using both generic questionnaires and to investigate correlations between these two clinical variables.

\section{Materials and Methods}

\section{Type of Study}

We conducted a sectional, descriptive and analytical study with a sample composed of 93 patients who were attended in the outpatient clinic of Knee Surgery Service of the Hospital in the period from December 2012 to May 2013. The individuals were recruited by non-probabilistic sampling of the sequential type from among those who met the inclusion criteria of the study.

\section{Sampling and Ethical Aspects}

The inclusion criteria were: patients with a diagnosis of (unilateral or bilateral) osteoarthritis of the knee and age between 40 and 70 years. Patients excluded were those with cognitive incapacity, who had undergone previous knee surgery, and persons who presented other associated diseases of the osteoarticular system (rheumatic diseases, osteometabolic diseases, etc.) and chronic and degenerative diseases that could interfere with the QOL and FI of the subjects, such as neoplasms, cardiopathies, and Parkinson's disease. The sample size was determined based on an estimate of $37 \%$ of prevalence for functional dependence ${ }^{10)}$, adopting an alpha of 0.05 and sampling error of $10 \%$. In this case, the estimated number was 93 individuals.

The patients were initially attended by an assistant physician and the procedure was adopted according to the clinical criteria of the service, and did not suffer any interference with the re- search protocol. After this, the subjects were referred for inclusion in the study. The patients who understood the research objectives and agreed to participate were included after signing the informed consent term. The research protocol was approved by the Research Ethics committee of the Hospital and in accordance with the Helsinki Declaration of 1975, revised in 2000.

\section{Procedures}

The subjects enrolled in the research were evaluated using data collected from their record charts and the specific instruments of the study. The general and clinical data were collected by a questionnaire containing the sociodemographic data of interest, such as gender, age, race, marital status, religion, profession, educational level, occupation, medical diagnosis and time of disease. After collection of these data, arthrosis of the knee was carefully evaluated and classified by an orthopedic specialist of the Brazilian Society of Knee Surgery. Osteoarthritis of the knee was stratified based on the radiographic status according the classification of Ahlbach ${ }^{13)}$, which is most widely used in the specialty of orthopedics. For this purpose, radiographs of the affected knee were taken with monopodal support in the anteroposterior and profile incidences. The knees were stratified according to the degree of arthrosis, ranging from grade I (slight arthrosis with joint space narrowing) up to grade $\mathrm{V}$ (severe arthrosis with joint subluxation), taking into account the most severely affected knee.

For evaluation of the patient's overall FI, the Barthel index was used $^{11,12,14)}$. This index evaluates the subject's level of independence for performing ten basic activities of daily living, namely, eating, personal hygiene, use of toilet, bathing, dressing and undressing, sphincter control, deambulation, transfer from chair to bed and climbing up and down stairs ${ }^{14)}$. The Barthel index is one of the most widely used generic rating scales for measurement of activity limitations in patients with neuromuscular and musculoskeletal conditions, as especially recommended for functional assessment of elderly people. The activities are assessed on a scale of four categories classified as 0 to 15 or 0 to 3 points, and the patients can score a maximum of 100 points in the former case or 20 points in the latter. Patients can be classified into the following categories: independent or functionally independent (20 points); slightly dependent (range, 15 to 19 points); moderately dependent (range, 10 to 14 points); and severely dependent (range, 0 to 9 points) ${ }^{11,12,14)}$.

Evaluation of the patient's QOL was measured by means of the SF-36 questionnaire and the SF-36 ${ }^{15)}$. The instrument is composed of 36 items grouped into 8 domains: functional capacity (or physical function), physical aspects (or role-physical), pain (or 
bodily pain), general state of health, vitality, social aspects (or social function), emotional aspects (or role-emotional) and mental health. For each subject and for each of the eight domains, a score was obtained by applying a measurement scale with values ranging from 0 (most compromised) to 100 (uncompromised) ${ }^{15)}$.

Both the Barthel index and the SF-36 were applied by the researchers themselves, in the form of a structured interview, with the questions being read by the researcher, seeking to obtain maximum responses.

\section{Statistical Analysis}

The data obtained were presented in frequency distribution tables in the case of discrete variables and in mean and standard deviation in the case of continuous variables. For the purpose of analysis, the followings were considered independent variables: age, gender, marital status, religion, race, educational level, main occupation, profession and medical diagnosis. The variable adopted as dependent was the QOL measured by the SF-36 questionnaire. The association between the dependent variable and independent variables was evaluated by the Chi-square test in the

Table 1. Sociodemographic Characteristics of the Patients

\begin{tabular}{lc}
\hline \multicolumn{1}{c}{ Variable } & No. of patients $(\%)$ \\
\hline Gender & $69(74.2)$ \\
Female & $24(25.8)$ \\
Male & \\
Marital status & $42(45.2)$ \\
Married & $28(30.1)$ \\
Single & $23(24.7)$ \\
Others & \\
Religion & $67(72.0)$ \\
Catholic & $26(27.9)$ \\
Others & \\
Schooling & $40(45.4)$ \\
Primary & $32(36.3)$ \\
Secondary & $16(18.2)$ \\
Superior & \\
Race & $23(24.7)$ \\
White & $36(38.7)$ \\
Black & $34(36.6)$ \\
Mulatto & \\
Work status & \\
Working & \\
Retired & \\
\hline & \\
\hline & \\
\hline & \\
\hline & \\
\hline
\end{tabular}

case of discrete variables (or Fisher test when pertinent) or by the $t$-test in the case of continuous variables. To evaluate the correlation between the QOL and the variables of interest, the Pearson correlation coefficient was used. In all cases, an alpha of 0.05 was adopted for statistical significance.

\section{Results}

The mean age of the patients was 60 years (range, 54.0 to 69.5 years) with 69 (74.2\%) subjects being the female gender and 24 (25.8\%) the male gender. With regard to the marital status, 42 (42.5\%) individuals were married. Sixty-seven (72\%) patients belonged to the Catholic religion and $40(45.4 \%)$ had an educational level of up to complete primary schooling. Thirty-six (38.7\%) patients were black, followed by mulattoes (34 patients, 36\%) and Caucasians (23 patients, 24.7\%) (Table 1).

Regarding the FI of the patients, 32 (34.3\%) were found to be independent, with the remaining patients categorized as either slightly dependent (53 patients, $57.1 \%$ ) or moderately dependent (8 patients, 8.6\%). None of them had independence alterations related to their sphincter control (bladder or bowel). Of the total sample, 64 (68.8\%) were retired and 29 (31.1\%) were still actively working. Only 20 patients had unilateral disease. With respect to the degree of arthrosis, the patients were distributed as fol-

Table 2. Functional Independence, Work Status, Time of Diagnosis, and Osteoarthritis Classification of the Patients

\begin{tabular}{lc}
\hline \multicolumn{1}{c}{ Variable } & No. of patients (\%) \\
\hline Functional independence & $32(34.3)$ \\
Independent & $53(57.1)$ \\
Light dependence & $8(8.6)$ \\
Moderate dependence & \\
Age (yr) & $60(54.0-69.5)$ \\
Mean (range) & $60(64.5)$ \\
$>65$-year-old & $33(35.4)$ \\
$<65$-year-old & \\
Time of diagnosis (yr) & $5.0(2.0-10.0)$ \\
Mean (range) & \\
Osteoarthritis & $8(8.6)$ \\
Grade I & $19(20.4)$ \\
Grade II & $17(18.3)$ \\
Grade III & $30(32.3)$ \\
Grade IV & $19(20.4)$ \\
Grade V &
\end{tabular}


Table 3. Comparison between Functional Independence and Quality of Life in Patients with Knee Osteoarthritis

\begin{tabular}{lccc}
\hline \multirow{2}{*}{ SF-36 domains s } & \multicolumn{2}{c}{ Functional independence (range) } & \multirow{2}{*}{ p-value } \\
\cline { 2 - 3 } & Dependent & Independent & \\
\hline Physical function & $20.0(12.5-35.0)$ & $60.0(35.0-88.8)$ & 0.001 \\
Role-physical & $0.0(0.0-25.0)$ & $31.2(0.0-93.8)$ & 0.005 \\
Bodily pain & $20.0(20.0-35.5)$ & $41.0(21.0-69.5)$ & 0.001 \\
General health & $52.0(40.0-62.0)$ & $62.0(52.0-77.0)$ & 0.004 \\
Vitality & $45.0(27.5-60.0)$ & $60.0(40.0-75.0)$ & 0.009 \\
Social function & $37.5(25.0-62.5)$ & $68.8(25.0-87.5)$ & 0.010 \\
Role-emotional & $0.0(0.0-66.6)$ & $66.6(0.0-100.0)$ & 0.002 \\
Mental health & $52.0(34.0-74.0)$ & $78.0(57.0-91.0)$ & 0.001 \\
\hline
\end{tabular}

SF-36: Medical Outcomes Study 36-Item Short Form Health Survey.

Table 4. Correlation between Functional Independence and Quality of Life in Patients with Knee Osteoarthritis

\begin{tabular}{lcr}
\hline \multicolumn{1}{c}{ Variable } & Correlation coeficient & p-value \\
\hline Functional independence vs. SF-36 & \\
Physical function & 0.609 & $<0.001$ \\
Role-physical & 0.304 & 0.006 \\
Bodily pain & 0.410 & $<0.001$ \\
General health & 0.373 & 0.001 \\
Vitality & 0.342 & 0.002 \\
Social function & 0.261 & 0.019 \\
Role-emotional & 0.248 & 0.026 \\
Mental health & 0.402 & $<0.001$ \\
\hline
\end{tabular}

SF-36: Medical Outcomes Study 36-Item Short Form Health Survey.

lows: grade I, 8 (8.6\%); grade II, 19 (20.4\%); grade III, 17 (18.3\%); grade IV, 30 (32.3\%); and grade V, 19 (20.4\%). With regard to the time of arthrosis, the sample presented a mean time of 5 years, ranging in the interval from 2 to 10 years (Table 2).

There was no significant correlation of FI with age $(\mathrm{p}=0.114)$, bilaterality ( $\mathrm{p}=0.342$ ), and time of diagnosis ( $\mathrm{p}=0.092)$. However, there was a significant correlation between the degree of osteoarthritis and FI $(\mathrm{p}=0.012)$. Therefore, $77.8 \%$ of grade I patients were completely independent while only $33.3 \%$ of grade IV patients were considered to be in the same level. When compared, individuals considered dependent or independent presented statistically significant difference in all of the functional domains of the QOL scale of the SF-36 (Table 3). From these data, a correlation was found between the QOL, FI and grade of arthrosis. The correlation between FI and QOL was shown to be statistically significant with respect to the all domains of the SF-36. The strongest correlation with FI was noted for functional capacity
Table 5. Correlation between Knee Osteoarthritis Classification ${ }^{13)}$ and Quality of Life in Patients with Knee Osteoarthritis

\begin{tabular}{lcc}
\hline \multicolumn{1}{c}{ Variable } & Correlation coeficient & p-value \\
\hline Osteoarthritis classification vs. SF-36 & \\
Physical function & -0.166 & 0.140 \\
Role-physical & -0.181 & 0.108 \\
Bodily pain & -0.008 & 0.942 \\
General health & -0.034 & 0.763 \\
Vitality & -0.154 & 0.173 \\
Social function & +0.108 & 0.342 \\
Role-emotional & -0.093 & 0.413 \\
Mental health & -0.027 & 0.815 \\
\hline
\end{tabular}

SF-36: Medical Outcomes Study 36-Item Short Form Health Survey.

(physical function), followed by the domains of pain (bodily pain) and mental health (Table 4). With regard to the degree of arthrosis, the correlation was not statistically significant (Table 5).

\section{Discussion}

Our results demonstrated that the functionality of the patient with osteoarthritis of the knee was associated with low QOL in all domains of the SF-36 questionnaire especially in pain (bodily pain), functional capacity (physical function) and mental health domains. There was also statistically significant positive correlation between the variable of FI and all the QOL domains; the more the FI was impaired, the lower the QOL was. The highest correlations were observed in pain, functional capacity, and mental health domains. The severity of osteoarthritis, however, was not correlated with significant loss of QOL in any domain.

Osteoarthrosis of the knees is considered a chronic disease capable of producing remarkable functional limitations associated with pain. These two elements would consequently be capable of explaining reduced QOL in patients who present with osteoarthritis of the knee ${ }^{6,716-18)}$. The elderly are especially susceptible to a reduction in FI. Most of the large studies have systematically confirmed the association between the loss of QOL and osteoarthrosis of the knee, and also shown a relationship with pain and functional capacity domains ${ }^{6,719)}$.

Seventy-four percent of our patients were women with a median age of 60 years. It has been known that sarcopenia and low levels of physical activity lead to weakness and imbalance of the knee joint from the age of 60 years, affecting the individual's functional activity ${ }^{8,10,20)}$. Our findings, therefore, confirm the fact that patients with osteoarthrosis of the knee (women over the age of 65 years in our study) with pain and impaired functional 
capacity suffer from diminished QOL.

It is estimated that $64 \%$ of the patients with OA suffer from important pain in the knees, and $98.6 \%$ of these patients present with morning rigidity with accentuated difficulties in performing the activities of daily life ${ }^{21)}$. Alves and Bassitt ${ }^{10)}$ found that half of their patients with OA reported intense or very intense pain when climbing up and down stairs and performing domestic tasks; a major portion of their patients complained of rigidity associated with moderate difficulty of physical function. They also described that the greatest reductions in QOL were strongly associated with the loss of $\mathrm{FI}^{10)}$. Watanabe et al. ${ }^{17)}$ demonstrated that worsening mobility of the knee is a factor sufficient to cause a reduction in the physical function of patients with OA, with the lower level of physical activity and higher energy expenditure also being the main factors negatively correlated with the QOL ${ }^{17)}$. In our study, radiographic severity was not correlated with QOL or with the FI of patients. Although our finding is in disagreement with those of some studies that have evaluated these two variables $^{22,23)}$, the majority of authors agree that pain and incapacity are the main predictors of QOL irrespective of radiographic severity $^{22-24)}$. In the study of Creamer et al. ${ }^{22)}$, there was no correlation between FI and the degree of OA or severity of radiographic alterations; however, there was a correlation between FI and pain, with pain being the only isolated predictive factor for FI in the multivariate model.

The QOL has become relevant and begun to influence treatment decisions and therapeutic procedures in patients with $\mathrm{OA}$ of the knee. Pain relief, restoration of function and improvement in general QOL have become parts of the evaluation of OA as indicators of the efficiency and impacts of the treatment of patients with severe disease $\mathrm{e}^{20,22,23)}$. However, only relief of pain has been reported as a consistent predictor associated with improvement of QOL in multivariate analysis ${ }^{25}$, which could have decreased the importance of improvement in mobility, function capacity, and ability for daily living activities.

The current study points out that FI continues to represent a crucial role for patients with knee OA. The strong correlation between FI and pain shows that these two variables are strongly associated. Our findings demonstrated that functional capacity is even more important than the severity of osteoarthritis. Therefore, mobility and function should be included in the evaluation of patient satisfaction with osteoarthritis treatment, especially in severe cases subjected to total knee arthroplasty.

In our study, we used two generic questionnaires, one for the assessment of the QOL and the other for functional limitation. The fact of not using specific questionnaires may have dimin- ished the sensitivity to detect specific impacts of osteoarthrosis of the knee. In spite of this limitation, the majority of studies that have evaluated incapacity in OA of the knee have also used generic questionnaires and reflected the general quality of health or health-related QOL to a greater extent than the patient's function. In contrast, in our study, we used an associated questionnaire with a specific focus on the overall function of the individuals. The sample size may not have been adequate for the analysis of subgroups of QOL, or adequate for the multiple analyses that would be possible by the diverse independent variables such as underlying disease. Studies with a larger sample size must be the objective of other researches with this specific purpose.

We may say that the original contribution of our study to osteoarthrosis of the knee was the fact that we conducted an in-depth evaluation of the association and correlation existent between QOL and FI. Our data showed a strong association between QOL and functional capacity, also demonstrating a negative correlation between the FI and all the QOL domains. These findings emphasize the clinical significance and importance of functional evaluation in patients suffering from knee osteoarthritis.

\section{Conclusions}

We conclude that general FI is directly associated with QOL; therefore, knee osteoarthritis has a large negative impact on both patient's daily living activities and their expectations on the treatment results. The strong correlation between these two variables also demonstrates that FI positively affects QOL.

\section{Conflict of Interest}

No potential conflict of interest relevant to this article was reported.

\section{References}

1. Corti MC, Rigon C. Epidemiology of osteoarthritis: prevalence, risk factors and functional impact. Aging Clin Exp Res. 2003;15:359-63.

2. Dillon CF, Rasch EK, Gu Q, Hirsch R. Prevalence of knee osteoarthritis in the United States: arthritis data from the Third National Health and Nutrition Examination Survey 1991-94. J Rheumatol. 2006;33:2271-9.

3. Cecchi F, Mannoni A, Molino-Lova R, Ceppatelli S, Benvenuti E, Bandinelli S, Lauretani F, Macchi C, Ferrucci L. Epidemiology of hip and knee pain in a community based 
sample of Italian persons aged 65 and older. Osteoarthritis Cartilage. 2008;16:1039-46.

4. Lawrence RC, Felson DT, Helmick CG, Arnold LM, Choi H, Deyo RA, Gabriel S, Hirsch R, Hochberg MC, Hunder GG, Jordan JM, Katz JN, Kremers HM, Wolfe F; National Arthritis Data Workgroup. Estimates of the prevalence of arthritis and other rheumatic conditions in the United States: part II. Arthritis Rheum. 2008;58:26-35.

5. de Rezende MU, de Campos GC, Pailo AF. Current concepts in osteoarthritis. Acta Ortop Bras. 2013;21:120-2.

6. Dominick KL, Ahern FM, Gold CH, Heller DA. Health-related quality of life among older adults with arthritis. Health Qual Life Outcomes. 2004;2:5.

7. Mili F, Helmick CG, Moriarty DG. Health related quality of life among adults reporting arthritis: analysis of data from the Behavioral Risk Factor Surveillance System, US, 199699. J Rheumatol. 2003;30:160-6.

8. Sutbeyaz ST, Sezer N, Koseoglu BF, Ibrahimoglu F, Tekin D. Influence of knee osteoarthritis on exercise capacity and quality of life in obese adults. Obesity (Silver Spring). 2007; 15:2071-6.

9. Aglamis B, Toraman NF, Yaman H. Change of quality of life due to exercise training in knee osteoarthritis: SF-36 and WOMAC. J Back Musculoskelet Rehabil. 2009;22:43-8.

10. Alves JC, Bassitt DP. Quality of life and functional capacity of elderly women with knee osteoarthritis. Einstein (Sao Paulo). 2013;11:209-15.

11. Lopez-Liria R, Padilla-Gongora D, Catalan-Matamoros D, Rocamora-Perez P, Perez-de la Cruz S, Fernandez-Sanchez M. Home-Based versus Hospital-Based Rehabilitation Program after Total Knee Replacement. Biomed Res Int. 2015; 2015:450421.

12. Sastre S, Sanchez MD, Lozano L, Orient F, Fontg F, Nunez M. Total knee arthroplasty: better short-term results after subvastus approach: a randomized, controlled study. Knee Surg Sports Traumatol Arthrosc. 2009;17:1184-8.

13. Ahlback S. Osteoarthrosis of the knee: a radiographic investigation. Acta Radiol Diagn (Stockh). 1968;Suppl 277:7-72.

14. Mohammad Hosseinzadeh F, Hosseinzadeh-Attar MJ, Yekaninejad MS, Rashidi B. Effects of selenium supplementation on glucose homeostasis and free androgen index in women with polycystic ovary syndrome: a randomized, double blinded, placebo controlled clinical trial. J Trace Elem
Med Biol. 2016;34:56-61.

15. Ware JE Jr, Sherbourne CD. The MOS 36-item short-form health survey (SF-36). I: conceptual framework and item selection. Med Care. 1992;30:473-83.

16. Heijink A, Gomoll AH, Madry H, Drobnic M, Filardo G, Espregueira-Mendes J, Van Dijk CN. Biomechanical considerations in the pathogenesis of osteoarthritis of the knee. Knee Surg Sports Traumatol Arthrosc. 2012;20:423-35.

17. Watanabe H, Urabe K, Takahira N, Ikeda N, Fujita M, Obara S, Hendona T, Aikawa J, Itoman M. Quality of life, knee function, and physical activity in Japanese elderly women with early-stage knee osteoarthritis. J Orthop Surg (Hong Kong). 2010;18:31-4.

18. Laslett LL, Quinn SJ, Winzenberg TM, Sanderson K, Cicuttini F, Jones G. A prospective study of the impact of musculoskeletal pain and radiographic osteoarthritis on health related quality of life in community dwelling older people. BMC Musculoskelet Disord. 2012;13:168.

19. Adegoke B, Alao R, Odole A. Comparison of health-related quality of life of Nigerian patients with knee osteoarthritis and age-matched controls. J Phys Ther. 2012;6:13-20.

20. Debi R, Mor A, Segal O, Segal G, Debbi E, Agar G, Halperin N, Haim A, Elbaz A. Differences in gait patterns, pain, function and quality of life between males and females with knee osteoarthritis: a clinical trial. BMC Musculoskelet Disord. 2009;10:127.

21. Majani G, Giardini A, Scotti A. Subjective impact of osteoarthritis flare-ups on patients' quality of life. Health Qual Life Outcomes. 2005;3:14.

22. Creamer P, Lethbridge-Cejku M, Hochberg MC. Factors associated with functional impairment in symptomatic knee osteoarthritis. Rheumatology (Oxford). 2000;39:490-6.

23. van Baar ME, Dekker J, Lemmens JA, Oostendorp RA, Bijlsma JW. Pain and disability in patients with osteoarthritis of hip or knee: the relationship with articular, kinesiological, and psychological characteristics. J Rheumatol. 1998;25:125-33.

24. Hopman-Rock M, Odding E, Hofman A, Kraaimaat FW, Bijlsma JW. Physical and psychosocial disability in elderly subjects in relation to pain in the hip and/or knee. J Rheumatol. 1996;23:1037-44.

25. Kim TK, Chang CB, Kang YG, Kim SJ, Seong SC. Causes and predictors of patient's dissatisfaction after uncomplicated total knee arthroplasty. J Arthroplasty. 2009;24:263-71. 